\title{
Tratamento de aneurisma da artéria renal por embolização e técnica de remodelamento de colo: relato de caso
}

\author{
Endovascular treatment of renal artery utilizing embolization and aneurism neck \\ remodeling technique: case report
}

Luis Carlos Mendes de Brito', João de Toledo Martins², Eliane Passos 33 , Amanda Jardim dos Santos', Rodrigo Assad Diniz da Gama 4 , Gabriela Ximenes Furlani ${ }^{5}$

\begin{abstract}
Resumo
O tratamento endovascular dos aneurismas de artéria renal tem sido descrito como alternativa à cirurgia convencional. Relatamos o caso de uma paciente com um aneurisma de artéria renal complexo à direita que apresentava hipertensão arterial de difícil controle. O tratamento endovascular foi realizado com a técnica de remodelagem de colo (técnica de Moret), ou técnica de embolização assistida por balão. A paciente obteve normalização da pressão arterial após o procedimento sem recidiva dos sintomas ou necessidade do uso de drogas anti-hipertensivas.
\end{abstract}

Palavras-chave: Aneurisma; artéria renal; procedimentos endovasculares.

\begin{abstract}
Endovascular treatment of renal artery aneurysms has been described as an alternative to conventional surgery. We report the case of a patient with complex renal artery aneurysm on the right kidney who had hard-to-control arterial hypertension. Endovascular treatment was performed with the aneurysm neck remodeling technique (Moret technique), or balloon-assisted coil embolization. The patient achieved blood pressure normalization after the procedure without recurrence of symptoms or need for antihypertensive drugs.
\end{abstract}

Keywords: Aneurysm; renal artery; endovascular procedures.

\section{Introdução}

Os aneurismas de artéria renal (AAR) constituem $22 \%$ dos aneurismas viscerais ${ }^{1}$ e $1 \%$ de todos os aneurismas ${ }^{2,3}$. O tratamento cirúrgico é frequentemente complexo, principalmente se os aneurismas forem intraparenquimatosos ou acometerem a bifurcação da artéria renal. A conduta mais comum é a aneurismectomia combinada com a arterioplastia ou ponte aorto-renal. A aneurismectomia pode ser realizada com sucesso em mais de $95 \%$ dos casos de aneurismas extraparenquimatosos, enquanto que, em certos tipos de aneurismas intraparenquimatosos ou que acometem a bifurcação da artéria renal, a aneurismectomia pode levar à nefrectomia parcial ou total. Recentemente, o tratamento endovascular tem sido empregado, principalmente para os aneurismas complexos, evitando infartos segmentares renais e nefrectomias ${ }^{4,5}$. $\mathrm{O}$ advento de microcateteres, fios-guias e diversos materiais embólicos, usados originalmente na Neurorradiologia intervencionista, possibilitou uma embolização mais seletiva, mesmo em lesões mais complexas de artéria renal e de colo largo ${ }^{6}$.

A técnica de Moret ou técnica de remodelagem de colo consiste na embolização assistida por balão. Trata-se de técnica amplamente utilizada no tratamento de aneurismas intracranianos de colo largo e que, atualmente, tem sido realizada com sucesso no tratamento de aneurismas complexos da artéria renal'.

'Radiologista Intevencionista e Cirurgião Vascular do Hospital Santa Lucinda, do Centro de Ciências Médicas e da Saúde da Pontifícia Universidade Católica de São Paulo (PUC-SP), do Serviço de Hemodinâmica e Arteriografia Digital do Hospital Evangélico de Sorocaba e do Hospital da Unimed de Sorocaba, Sorocaba (SP), Brasil.

${ }^{2}$ Radiologista Intervencionista do Hospital Santa Lucinda do Centro de Ciências Médicas e da Saúde da PUC-SP, do Serviço de Hemodinâmica e Arteriografia Digital do Hospital Evangélico de Sorocaba e do Hospital da Unimed de Sorocaba, Sorocaba (SP), Brasil.

${ }^{3}$ Clínica Geral e Cardiologista do Hospital Unimed de Sorocaba, Sorocaba (SP), Brasil.

${ }^{4}$ Acadêmica do curso de Medicina da Faculdade de Ciências Médicas e da Saúde da PUC-SP, Sorocaba (SP), Brasil.

${ }^{5}$ Acadêmica do Curso de Medicina da Universidade Cidade de São Paulo (UNICID), São Paulo (SP), Brasil.

Não foram declarados conflitos de interesse associados à publicação deste artigo.

Submetido em: 11.08.2010. Aceito em: 18.05.2011

J Vasc Bras. 2011;10(2):181-184. 
Relatamos o caso de uma paciente em que se realizou o tratamento endovascular com técnica de microcatéter assistida por balão com colocação de microcoils em aneurisma saculiforme da bifurcação da artéria renal direita, com preservação do fluxo sanguíneo renal.

\section{Descrição do caso}

Mulher branca, 56 anos, encaminhada com quadro de hipertensão arterial de difícil controle há 26 anos, mesmo com o uso de 4 drogas anti-hipertensivas e tentativas de troca de drogas sem melhora. Apresentava diabetes melito e obesidade (índice de massa corporal $>30$ ). No último ano, apresentou piora progressiva e descontrole da doença, com quadro de cefaleia noturna e hipertensão arterial grave, indicando-se, então, tratamento intervencionista. A pressão arterial no pré-operatório era de 220/110 (medida em membro superior direito e esquerdo). Medicamentos em uso: irbesartana $300 \mathrm{mg}$, hidroclorotiazida $25 \mathrm{mg}$, atenolol $50 \mathrm{mg}$, enalapril $10 \mathrm{mg}$, metformina $850 \mathrm{mg}$, glimeperida $2 \mathrm{mg}$. Realizou-se ultrassom Doppler, sugerindo estenose maior que $70 \%$ em artéria renal direita. A arteriografia demonstrou aneurisma saculiforme de $9 \mathrm{~mm}$ de diâmetro em artéria renal direita na sua bifurcação (Figura 1). Apresentava creatinina sérica de $1,2 \mathrm{mg} / \mathrm{dL}$, proteinúria de $137 \mathrm{mg}$, ureia $41 \mathrm{mg} / \mathrm{dL}$, clearance de creatinina $76 \mathrm{~mL} / \mathrm{min}$.

Decidiu-se realizar embolização com a técnica de remodelamento de colo (técnica de Moret). A artéria renal foi cateterizada com cateter guia RDC 7F e, através dele, foram passadas duas microguias 0,014 inc, uma para a artéria de maior calibre, que direcionou e posicionou o balão para o remodelamento do colo (Hiperglide EV3 de $6 \mathrm{~mm}$ ), e a outra foi posicionada dentro do saco aneurismático e utilizada para levar o microcateter Excel 10 para dentro do saco. O balão foi insuflado até $4 \mathrm{~mm}$ e, em seguida, através do microcateter, preencheu-se o saco aneurismático

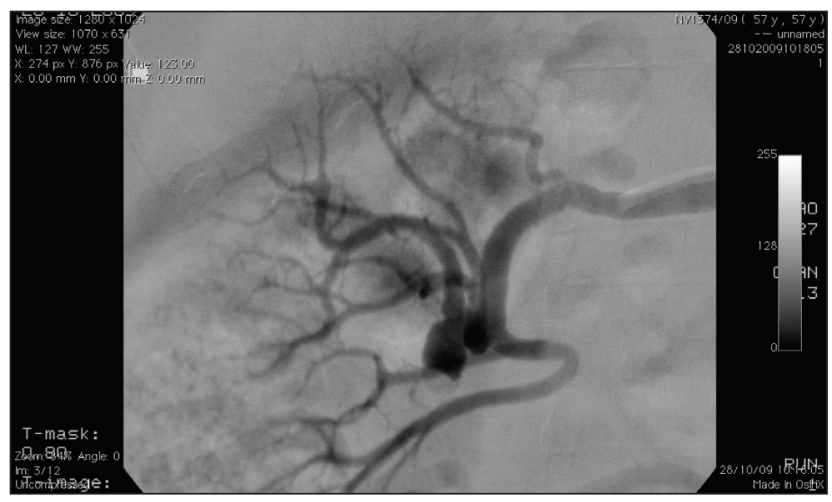

Figura 1 - Arteriografia renal direita: aneurisma de artéria renal complexo. com 6 micromolas de largagem controlada (GDC Boston Scientific, Natick, MA, USA) (Figura 2). Após o preenchimento do aneurisma, o balão foi esvaziado e retirado. Realizou-se angiografia de controle após o procedimento, que demonstrou a exclusão do saco aneurismático, com a artéria renal e seus ramos pérvios e com bom nefrograma, sem áreas de isquemia (Figura 3). Imediatamente após a realização do procedimento, a paciente já se encontrava

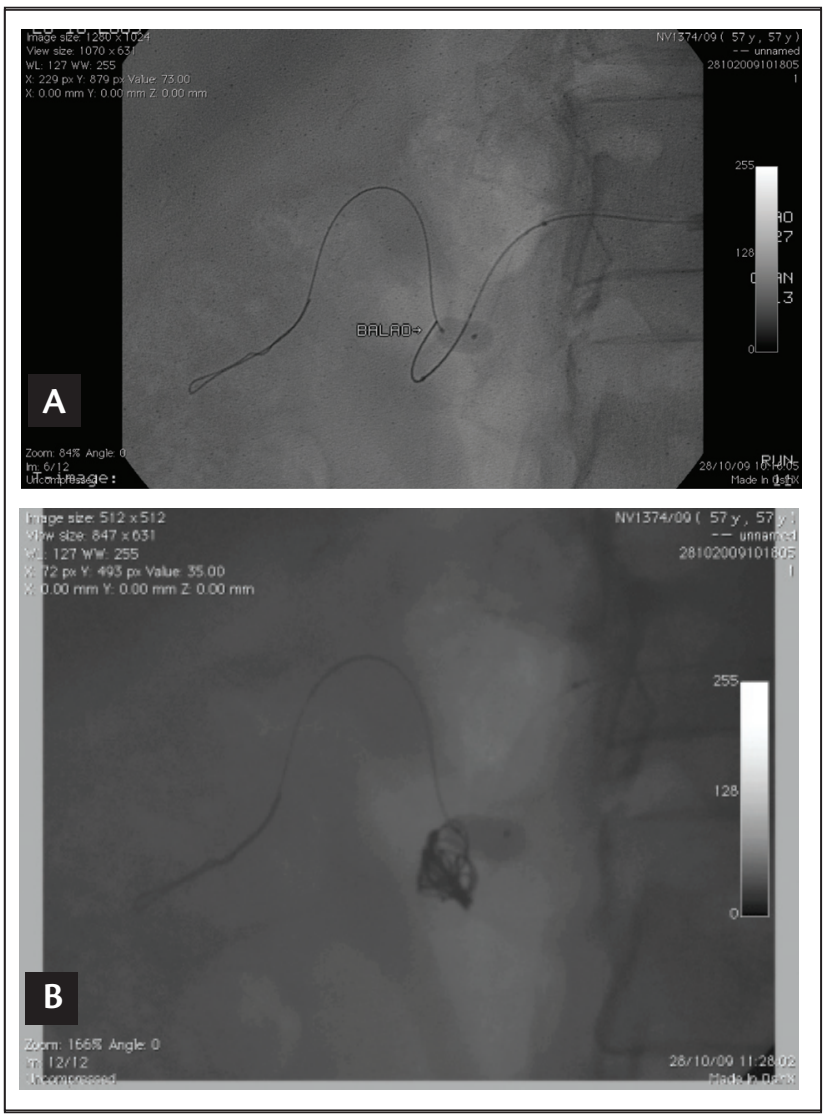

Figura 2 - (A) Posicionamento do balão. (B) remodelamento do colo com balão e preenchimento do saco aneurismático com as micromolas.

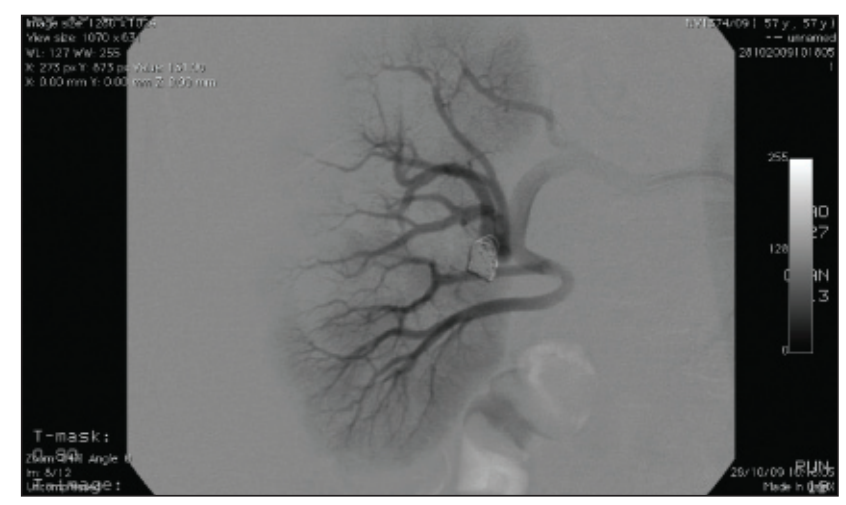

Figura 3 - Exclusão do saco aneurismático sem evidência de áreas de isquemia. 
normotensa. Durante um ano de acompanhamento clínico, até este momento, não houve recidivas dos sintomas, nem a necessidade do uso de anti-hipertensivos.

\section{Discussão}

Os AAR, apesar de representarem patologia pouco usual, constituem $22 \%$ dos aneurismas viscerais. Seu achado é frequentemente incidental em estudos por imagem durante investigação de doenças como a hipertensão, especialmente. Esses aneurismas são mais prevalentes no sexo feminino, a maioria é sacular e não calcificada, ocorrendo com maior frequencia na bifurcação da artéria renal principal ${ }^{7}$. Tem como causas: doenças degenerativas (aterosclerose e displasia fibromuscular), vasculites, trauma, origem idiopática, neoplasias, iatrogenia aneurismas micóticos e outras causas em menor frequencia. Os AAR associam-se à hipertensão arterial em até $70 \%$ dos casos ${ }^{8}$, podendo apresentar origem renovascular devido a certas causas, dentre elas, embolização, compressão extrínseca e tortuosidade da artéria adjacente $\mathrm{e}^{9,10}$. Outras complicações associadas aos AAR consistem em dissecção, infarto renal e fístula arteriovenosa. A indicação do tratamento deve levar em consideração a idade do paciente, sexo, idade fértil para as mulheres, presença de sintomas, hipertensão grave associada, características anatômicas do aneurisma, ruptura, tamanho, expansão e embolização distal. Frequentemente, aneurismas maiores que $20 \mathrm{~mm}$ de diâmetro têm sido indicados para o tratamento cirúrgico ou endovascular; entretanto, há relatos de ruptura em aneurismas com diâmetros inferiores ${ }^{8,11-14}$.

Devido ao fato de os AAR terem uma tendência de ocorrer na bifurcação da artéria renal principal, a oclusão da artéria renal durante procedimento cirúrgico convencional ou utilização de endoprótese pode levar a infarto significativo do parênquima renal. Com o desenvolvimento de microcateteres e microguias, utilizados originalmente em Neurorradiologia, aneurismas saculares complexos podem ser excluídos seletivamente e com maior segurança, sem o comprometimento do parênquima renal.

A técnica de remodelamento do colo, assistida por balão, foi descrita por Moret et al. ${ }^{11}$ Consiste no uso de microcateter com balão, o qual oclui temporariamente o colo aneurismático durante a colocação das micromolas, funcionando como barreira contra a migração para a artéria adjacente, além de proporcionar estabilidade ao microcateter.

Abath et al. ${ }^{6}$ descreveram a técnica assistada por balão e classificaram os AAR de acordo com as melhores alternativas para o tratamento endovascular. Na classificação, o tipo I (aneurismas saculares da artéria renal principal) teria como indicação o implante de stent recoberto ou com colocação de micromolas; o tipo II (aneurismas da bifurcação da artéria renal), a liberação de micromolas por meio da técnica de remodelagem, complementadas ou não com agentes embólicos líquidos, e o tipo III (aneurismas de pequenos ramos segmentares intraparenquimatosos), a oclusão da artéria nutridora com micromolas ou agentes líquidos ${ }^{6}$.

\section{Conclusão}

O tratamento endovascular de AAR a longo prazo ainda não está bem estabelecido, apesar da melhora dos controles pressóricos da paciente após a exclusão do aneurisma. Não se sabe sobre a possibilidade de recidiva dos sintomas ou expansão desses aneurismas. Entretanto, a técnica de micromolas associada à técnica assistida com balão está bem estabelecida na literatura e possui eficácia e segurança comprovadas no tratamento de aneurismas intracranianos, podendo ser facilmente adaptada para o tratamento bem sucedido de aneurismas da artéria renal complexos.

\section{Referências}

1. Deterling RA Jr. Aneurysm of the visceral arteries. J Cardiovasc Surg. 1971;12:309-22.

2. Post K, Hupp T, Roeren T, Brambs HJ, Allenberg JR, Kauffmann GW. Renal artery aneurysms. Radiologe. 1991;31:56-61.

3. Hageman JH, Smith RF, Szilagyi E, Elliott JP. Aneurysms of the renal artery: problems of prognosis and surgical management. Surgery. 1978;84:563-72.

4. Karkos CD, D'Souza SP, Thomson G), Chomal A, Matanhelia SS. Renal artery aneurysm: endovascular treatment by coil embolisation with preservation of renal blood flow. Eur J Vasc Endovasc Surg. 2000;19:214-6.

5. Cardozo MA, Lichtenfels E, Erling Jr N, Raupp E, Tarasconi DP. Tratamento endovascular de aneurisma da artéria renal por embolização com micromolas preservando o fluxo sangüíneo renal: relato de caso. I Vasc Bras. 2007;6:167-70.

6. Abath C, Andrade G, Cavalcanti D, Brito N, Marques R. Complex renal artery aneurysms: liquids or coils? Tech Vasc Interv Radiol. 2007;10:299-307.

7. Wajnberg E, Aquino D, Spilberg G. Experiência preliminar com o uso da técnica de "remodelagem de colo" para tratamento endovascular de aneurismas complexos da artéria renal. Radiol Bras. 2010;43:29-33.

8. Henke PK, Cardneau JD, Welling TH 3rd, Upchurch GR Jr, Wakefield TW, Jacobs LA, et al. Renal artery aneurysmsm: a 35-year clinical experience with 252 aneurysms in 168 patients. Ann Surg. 2001;234:454-62.

9. Tham G, Ekelund L, Herrlin K, Lindstedt EL, Olin T, Bergentz SE. Renal artery aneurysms. Natural history and prognosis. Ann Surg. 1983;197:348-52. 
10. Soussou ID, Starr DS, Lawrie GM, Morris GC Jr. Renal artery aneurysm. Long-term relief of renovascular hypertension by in situ operative correction. Arch Surg. 1979;114:1410-5.

11. Moret J, Pierot L, Boulin A, Castaings L, Rey A. Endovascular treatment of anterior communicating artery aneurysms using Guglielmi detachable coils. Neuroradiology. 1996;38:800-5.

12. English WP, Pearce JD, Craven TE, Wilson DB, Edwards MS, Ayerdi J, et al. Surgical management of renal artery aneurysms. I Vasc Surg. 2004; $40: 53-60$.

13. Martin RS $3^{\text {rd }}$, Meacham PW, Ditesheim JA, Mulherin JL Jr, Edwards WH. Renal artery aneurysm: selective treatment for hypertension and prevention of rupture. J Vasc Surg. 1989;9:26-34.

14. Hidai H, Kinoshita Y, Murayama T, Miyai K, Matsumoto A, Ide K, et al. Rupture of renal artery aneurysm. Eur Urol. 1985;11:249-53.
Correspondência: Amanda Jardim dos Santos Rua Padre Clemente Marton Segura, 94 - Cidade Nova CEP 15085-480 - São José do Rio Preto (SP), Brasil E-mail:amandajardimsa@gmail.com

Contribuições dos autores: Concepção e desenho do estudo: LCMB, JTM, AJS, RADG Análise e interpretação dos dados: LCMB, JTM, AJS, RADG Coleta de dados: $L C M B, E P, A J S$, RADG Redação do artigo: LCMB, AJS, RADG, GXF Revisão crítica do texto: LCMB, AJS, RADG Aprovação final do artigo*: LCMB, JTM, AJS, RADG, GXF Análise estatística: N/A Responsabilidade geral pelo estudo: LCMB Informações sobre financiamento: N/A *Todos os autores leram e aprovaram a versão submetida ao J Vasc Bras. 